\title{
Comment on Mycobacterium Chelonae Infection of the Buttocks Secondary to Lipofilling: A Case Report and Review of the Literature
}

\author{
Masoud Keikha ${ }^{1}$
}

Received: 11 October 2017/ Accepted: 12 October 2017/Published online: 3 November 2017

(c) Springer Science+Business Media, LLC and International Society of Aesthetic Plastic Surgery 2017

Level of Evidence $V$ This journal requires that authors assign a level of evidence to each article. For a full description of these Evidence-Based Medicine ratings, please refer to the Table of Contents or the online Instructions to Authors www.springer.com/00266.

\section{Dear Editor,}

Hammond et al. [1] recently published their report on Mycobacterium Chelonae Infection of the Buttocks Secondary to Lipofilling: A Case Report and Review of the Literature. Non-tuberculosis mycobacteria (NTM) are freeliving saprophytes that are isolated from environmental resources such as water, soil, dust, animals and dairy foods. Non-tuberculosis mycobacteria were not believed to be human pathogens before 1950s, but now it is clear that this group of bacteria can cause local cutaneous to serious disseminated infections in humans [2]. According to the American Thoracic Society (ATS) advice, clinically isolated NTM should be detected at the species level to determine the clinical implications, infection control, epidemiological study, and patient administration. NTM were identified using phenotypic tests (such as growth rate, macroscopic morphological features, growth at different temperatures, biochemical tests including Tween 80 hydrolysis, nitrate reduction, arylsulfatase, urease production, tellurite reduction, salt tolerance and semi-quantitative catalase production) and popular molecular methods including sequencing and PCR restriction fragment length

Masoud Keikha

Masoud.keykha90@gmail.com

1 Department of Microbiology, School of Medicine, Isfahan University of Medical Sciences, Isfahan, Iran polymorphism using $16 \mathrm{~S}$ rRNA, $h s p 65, r p o B$ and $16 \mathrm{~S}-23 \mathrm{~S}$ rRNA internal transcribed spacer (ITS) [3].

I ask the authors to attend to the following questions.

1. Please describe the mycobacterial isolation method, which was not stated in the report.

2. According to reports, other genuses in the Actinomycetes family such as Mycobacterium tuberculosis, Nocardia, Gordonia and Rhodococcus similar to NTM have the same phenotypic features [4]; please explain how Mycobacterium chelonae was detected to the species level.

\section{Compliance with Ethical Standards}

Conflict of interest The author declares that he has no conflicts of interest to declare.

\section{References}

1. Hammond SE, Al-Bayati A, Joumblat N, Salgado CJ (2017) Mycobacterium chelonae infection of the buttocks secondary to lipofilling: a case report and review of the literature. Aesthetic Plast Surg 41(5):1150-1154

2. Velayati AA, Farnia P, Mozafari M, Malekshahian D, Seif S, Rahideh S, Mirsaeidi M (2014) Molecular epidemiology of nontuberculous mycobacteria isolates from clinical and environmental sources of a metropolitan city. PLoS ONE 9(12):e114428

3. Hashemi-Shahraki A, Bostanabad SZ, Heidarieh P, Titov LP, Khosravi AD, Sheikhi N, Ghalami M, Nojoumi SA (2013) Species spectrum of nontuberculous mycobacteria isolated from suspected tuberculosis patients, identification by multi locus sequence analysis. Infect Genet Evolut 20:312-324

4. Saubolle MA, Sussland D (2003) Nocardiosis review of clinical and laboratory experience. J Clin Microbiol 41(10):4497-4501 